Pensamiento Crítico Vol. $19 \mathrm{~N}^{\circ} 1$, pp. 71-83

\title{
Retraso en la entrega de predios, un problema a resolver en las APP: efecto en los usuarios
}

Delay in delivery of land, a problem to solve in PPPs: effect on users Antonio Lama More ${ }^{1}$ Tito Vargas Príncipe ${ }^{2}$

\section{RESUMEN}

Este artículo aborda uno de los problemas usualmente recurrentes relacionado con la liberalización de terrenos en el Perú, enfocado en la concesión de la Red Vial №6 (concesión de carretera mediante una APP)

Es evidente que los problemas de entrega de terrenos son originados desde el diseño del contrato de concesión, debido a que el Estado, con el propósito de lograr el crecimiento de la inversión a través de proyectos de infraestructura, ha generado proyectos sin evaluar adecuadamente los estudios sobre la liberación de terrenos y su impacto en la ejecución de obras. El actual marco legal de expropiación en el Perú (que incluye el marco legal de liberalización de terrenos), no obstante haberce flexibilizado la norma, no logran administrar eficientemente la entrega de terrenos.

Bajo este contexto, se observa recurrentemente incumplimientos de entrega de terrenos en los plazos originalmente pactados por las partes en los proyectos de

1 Economista y Maestro en Ciencias, Profesor Principal de la Facultad de Ciencias Económicas

2 Economista de la UNMSM, con estudios de maestría en Economía de la Pontificia Universidad Católica del Perú y Analista Económico - Financiero de la Dirección General de Concesiones en Transportes del MTC. 


\section{Pensamiento Crítico Vol. 19. N I}

carreteras concesionadas. Estos incumplimientos tiene el principal efecto de retraso de las oportunidades de inversión establecidas en los contratos de concesión, causando retraso en los beneficios que los usuarios perciben sobre la ejecución de cada obra, en términos de dinero y tiempo (costo de viajes, accidentes costo operativo vehicular y otros)

Palabras clave: contrato de concesión, costo operativo vehicular, costo de oportunidad.

\section{ABSTRACT}

This article will address release issues of real estate in transports concessions in Peru, focused in the concession Red vial $N^{\circ} 6$ (highway concession by Public Private Partnership (PPP)).

It evidence that problems of real estate transfer are originated from the draft concession Agreement, because of Peruvian government, with the purpose of led to the growth the investment through of infrastructure projects, has generate politics without evaluate studies about land transfers and their impact in execution of works. Furthermore, the currently legal framework of expropriation in Perú (that include legal framework of release of real estate) doesn't allow at the government to manage efficiently real estate transfer.

In this context, recurrent breaches of delivery of land seen in the terms originally agreed by the parties to the concession road projects, these defaults have the main effect of delay the investment opportunities set out in the Concession Contract causing delays in the benefits that users perceive about the execution of such works in terms of money and time, for example (cost of trips, accidents, vehicle operating costs and others)

Keywords: Concession Contract, VehicleOperating Costs, Opportunity Cost

Clasificación del artículo3: JEL: L51, L91

\section{A manera de introducción}

3 Classification Journal of Economic Literature (JEL) 


\section{Antonio Lama More}

En la actualidad el Estado peruano, así como la mayoría de los Estados en la región, para cumplir con su rol de proveedor de servicios públicos ha optado por ejecutar los grandes proyectos de infraestructura pública vía la modalidad asociaciones públicas privadas (APP) o participación pública privada (PPI). Las reglas de ejecución de dichos proyectos se materializan por lo general a través de un contrato de concesión ${ }^{4}$. Dicho contrato tiene como fin último dotar de una infraestructura de calidad a los usuarios, que, para el caso del sector transportes, es proveer un aeropuerto, un puerto, un ferrocarril o una carretera dirigida a facilitar el bienestar de la sociedad y en particular de los usuarios, al disponer de servicios públicos que permitiesen atender los problemas económicos y sociales al interior de sus economías.

Al respecto, el sistema de concesiones peruano ha tenido un desarrollo adecuado que se evidencia en el índice Infrascope del $2012^{5}$, que ubica al Perú, de acuerdo a su evaluación, en el tercer lugar dentro de los países de Latinoamérica y el Caribe con mejor entorno ideal para el desarrollo de los proyectos APP dada la existencia de un desarrollo relativo de una institucionalidad y de un marco legal específico y adecuado.

Sin embargo, la experiencia nos lleva a verificar la suscripción de varias modificaciones a los términos contractuales es decir, de renegociaciones contractuales que han llevado a críticas no solo al sector, sino incluso a las políticas de renegociaciones. En particular, el presente artículo revisará una de las causas de dichas renegociaciones casi recurrentes en la mayorías de los contratos de APP. Nos referimos al desfase en la entrega de terrenos o áreas geográficas debidamente saneados, incidiendo en el oportuno y normal desarrollo de la ejecución de las obras, retrasando las inversiones programadas y los beneficios en el tiempo establecido de contar con la infraestructura concesionada en las oportunidades pactadas en el contrato, afectando el bienestar de los usuarios, la rentabilidad del concesionario y del Estado.

En este contexto, resulta relevante establecer los umbrales del presente artículo,

4 Las APP constituyen un mecanismo para promover la inversión privada, como parte de un proceso integral en el cual el Estado pasa a ejercer la función de un gran promotor y supervisor de las transacciones y las modalidades contractuales mediante las cuales se desarrollan: contratos de concesión, de operación, de gerencia, jointventure u otros permitidos por ley. La nota característica de las APP es la "mejor distribución de riesgos". El Estado "no se desprende", como en la privatización, "ni delega íntegramente" todos los riesgos al sector privado, como sucede en la concesión tradicional (o de "primera generación")

5 The Economist. "Evaluando el entorno para las asociaciones público privadas en América Latina y el Caribe". Infrascope 2012. Economist Intelligence Unit. 


\section{Pensamiento Crítico Vol. 19. N I}

que tiene como propósito esencial el análisis de la demora en la entrega de terrenos y sus efectos en el costo de oportunidad de los usuarios, quedando pendiente merituar para una siguiente oportunidad el análisis relacionado con sus efectos económicos y sociales sobre el concedente y el concesionario.

La evaluación de la incidencia en los usuarios tendrá como soporte referencial el análisis del contrato de concesión de la Red Vial $N^{\circ} 06$, teniendo como lógica ex positiva de lo general a lo particular, de tal forma que en la primera, se revisarán aspectos generales del contrato de concesión; en la segunda, se abordarán las consideraciones que habrían originado el problema de la entrega de terrenos en la oportunidades inicialmente previstas en el referido contrato de concesión; en la tercera, se abordará el objetivo del artículo estimando el efecto que habría ocasionado la demora de la entrega de terrenos en el bienestar de los usuarios, lo que permitirá, finalmente, arribar a las respectivas conclusiones y recomendaciones.

\section{Contrato de concesión de la Red Vial $\mathbf{N}^{\circ} 06$}

En nuestro país el Estado peruano ha suscrito 32 contratos de concesión con el sector privado, bajo la modalidad de APP6. Del total de dichos contratos, 15 son autosostenibles, los contratos autosostenibles generan ingresos que cubren el pago de las inversiones y los costos de operación y mantenimiento, así como la rentabilidad al privado y en muchos casos generan retribución al Estado; y 16 son cofinanciados, en este caso el Estado aporta un cofinanciamiento para cubrir los pagos de inversión operación y mantenimiento. Uno de los 28 contratos de concesión es el de la Red Vial $N^{\circ} 06$, el cual es de concesión autosostenible.

\subsection{Aspectos generales de la concesión de la Red Vial $N^{\circ} 06$}

La Concesión del Tramo Vial Puente Pucusana - Cerro Azul - Ica Red Vial tiene por objeto la construcción y explotación de la infraestructura de servicio público del tramo Vial Puente Pucusana - Cerro Azul - Ica, Red Vial, que incluye puestas a punto, construcción de tramos viales, operación, mantenimiento entre otras actividades detalladas en el contrato de concesión y sus respectivas adendas.

6 Las APP se basan en una alianza pública privada que viabilice una adecuada identificación, cuantificación y asignación de riesgos, tendentes a garantizar un desarrollo y operación eficiente del proyecto. 


\section{Antonio Lama More}

El Contrato de Concesión se suscribió el 20 de septiembre de 2005, con un periodo de vigencia de 30 años, el mismo que podría ampliarse a 32 años en el caso que la Segunda Etapa de construcción se inicie entre el año 17 y 19. El objeto de la Concesión es la construcción y explotación de 221,7 km de la carretera Panamericana Sur.

A continuación se describe las Obras Ejecutadas y las Obras por Ejecutar de acuerdo al cronograma de ejecución de Obras establecidas en el Anexo 2 del contrato de concesión:

\section{Obras ejecutadas}

A la fecha el Concesionario ha cumplido con las obligaciones contractuales referentes a Obras:

- Actividades Preparatorias

- $\quad$ Obras de la Primera Etapa (Autopista desde Cerro Azul hasta Chincha)

- Adelanto de algunas Obras de la Segunda Etapa (15 km aprox. del Módulo A hasta Chincha y el Puente Topará, las que fueron adelantadas dispuestas en la Adenda 4 del año 2011, con cargo a la Retribución del Estado)

\section{Obras por ejecutar:}

\section{Segunda etapa}

Las demás obras de la segunda etapa (concluir módulo a y el total del módulo B) de acuerdo al contrato de concesión se ejecutarían a partir de setiembre del 2016.

Con la ejecución de las obras del módulo A y B de la segunda etapa, se podrá contar con una "nueva autopista" desde Chincha Alta hasta San Andrés (Pisco) de una longitud de 41 kilómetros aproximadamente. 


\section{Pensamiento Crítico Vol. 19. $\mathrm{N}^{\circ}$ I}

\section{Tercera Etapa}

Finalmente las obras de la tercera etapa se gatilla cuando la vía registre 23 millones de ejes cobrables y, según lo establecido en el contrato de concesión, su ejecución es por acuerdo de las partes.

En el esquema se visualizan las etapas del contrato de concesión, para una mejor ilustración:

\section{Esquema gráfico de la concesión}

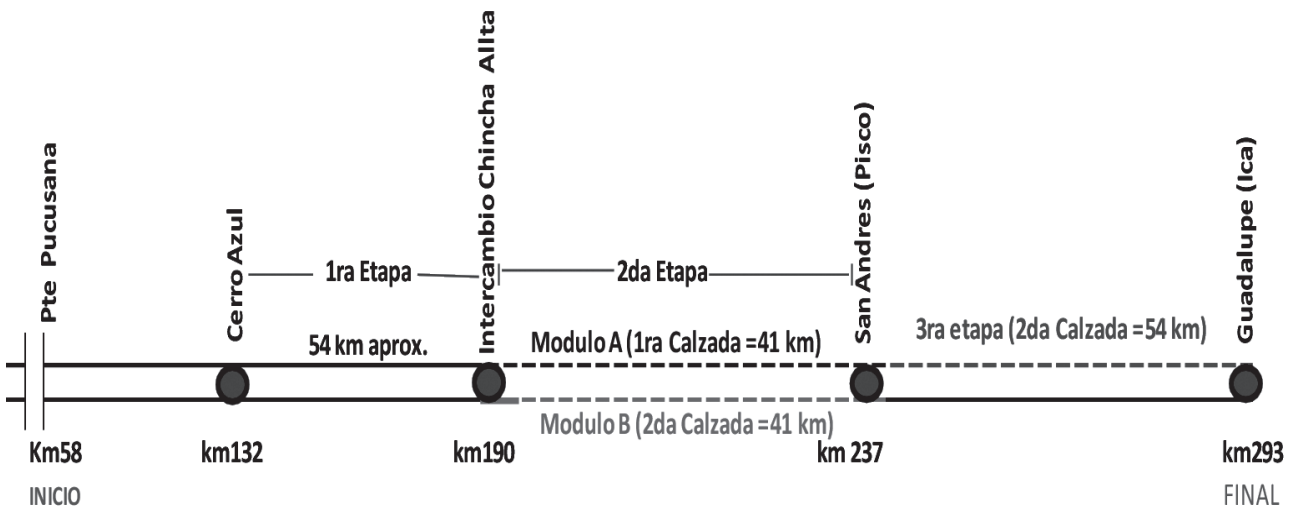

Fuente: Anexo II del Contrato de Concesión / Elaboración Propia

\subsection{Entrega de terrenos para la ejecución de la primer Etapa}

El contrato de concesión, en su Anexo II ${ }^{7}$, establece que la "Primera Etapa" de construcción "se ejecutará dentro de los dos (2) años posteriores a la entrega de los predios necesarios, pero en ningún caso el inicio de las Obras para esta etapa podrá ser antes del inicio del tercer año de la concesión, según lo señalado en la cláusula $5.2^{8}$ del contrato.

7 "Anexo II(...)

2. Primera Etapa

Se ejecutará dentro de los dos (2) años posteriores a la entrega de los predios necesarios, pero en ningún caso el inicio de las obras para esta etapa podrá ser antes del inicio del tercer año de la concesión, según lo señalado en las Cláusulas 5.2 y el presente anexo (...)"

8 Cláusula 5.2 “(...) 


\section{Antonio Lama More}

Dado que el contrato de concesión se suscribió en setiembre del 2005, el inicio de las obras de la primera etapa debió darse en setiembre del 2007, asumiendo que el Estado hubiese cumplido en entregar el terreno debidamente saneado.

Sin embargo, el Estado no cumplió con entregar en la oportunidad establecida en el contrato los predios necesarios para la ejecución de las obras de la primera etapa. El plazo para la entrega de terrenos para el Estado era de 540 días de suscrito el contrato ${ }^{9}$, es decir, el Estado tenía hasta marzo del 2007 para entregar los predios, pero este no pudo cumplir con dicha obligación. En consecuencia, las obras no pudieron darse inicio en la fecha prevista, setiembre del 2007.

Ante esta situación, las partes estimaron necesario suscribir una adenda al contrato de concesión para sincerar los plazos de entrega y para la postergación del inicio y final de las obras, tal como consta en la adenda $\mathrm{N}^{\circ} 2^{10}$.

Las partes acuerdan que aún en el caso en que el CONCEDENTE haya cumplido con la entrega de los predios necesarios para iniciar las obras de la primera etapa de conformidad con lo establecido en la presente cláusula, la SOCIEDAD CONCESIONARIA no se encontrará obligada a iniciar las mismas hasta el tercer año de la concesión, calculándose el plazo a que se refiere el anexo II para el inicio de las obras de la primera etapa a partir de eta fecha.(...)"

9 "Párrafo 10 de la cláusula 5.2

(i) no se produzca la entrega del ochenta por ciento $(80 \%)$ de los predios necesarios en términos del área total que constituye el derecho de vía, necesaria para que la SOCIEDAD CONCESIONARIA inicie los trabajos comprendidos en los sub- tramos de la primera etapa de construcción dentro del plazo de quinientos cuarenta (540) días calendario de suscrito el contrato o(...)"

10 Cláusula 3.2 de la adenda $\mathrm{N}^{\circ} 2$ (modifica la cláusula 5.2 del contrato) "(...)

Los predios urbanos o rurales afectados, necesarios para que la SOCIEDAD CONCESIONARIA inicie los trabajos comprendidos dentro de la primera etapa de construcción, deberán ser entregados por el CONCEDENTE, de acuerdo a lo siguiente:

a) Predios del sub tramo 3.- El $80 \%$ de los predios en términos del área total que constituye el derecho de vía serán entregados por el CONCEDENTE en un plazo máximo que vencerá el día 30 de mayo de 2009. El 20 \% restante deberá ser entregado a más tardar el día 30 de setiembre de 2009.

Subtramo 3: aquél comprendido entre los kms. 1+600 al 20+301 (Cerro Calavera - Pampa Clarita)

b) Predios del sub tramo 4: El $80 \%$ de los predios en términos del área total que constituye el derecho de vía, que corresponden el sub tramo 4, serán entregados por el CONCEDENTE en un plazo máximo que vencerá al día 30 de junio de 2009. El 20\% restante deberá ser entregado a más tardar el 30 de octubre de 2009.

Subtramo 4: aquél comprendido entre las progresivas 20+301 al 53+386 (Pampa Clarita - Intercambio Chincha Alta) (...."

Cláusula 3.3 de la adenda $N^{\circ} 2$ (modifica la Cláusula 6.1 del Contrato)”(...)

La SOCIEDAD CONCESIONARIA culminará las demás obras previstas en la primera etapa el día 31 de diciembre de 2010, (...)" 


\section{Pensamiento Crítico Vol. 19. $\mathrm{N}^{\circ}$ I}

Con dichas modificaciones y ampliaciones de plazo, las obras de la primera etapa de la Red Vial N 06 se culminaron recién en setiembre del 2011, observándose dos años de retraso de las inversiones. Lo señalado en este punto se resume en el siguiente cuadro:

\begin{tabular}{|l|c|c|}
\hline Conceptos & Contrato & Contrato + Adenda $\mathbf{N}^{\circ} \mathbf{0 2}$ \\
\hline Suscripción del Contrato & sep-05 & sep-05 \\
Plazo de entrega de terrenos & 540 & \\
Fecha límite para entrega de terrenos & mar-07 & \\
Inicio de Obras 1ra Etapa & sep-07 & jun-08 \\
Plazo de ejecución (años) & 2 & 3 \\
Final de la 1ra Etapa & sep-09 & sep-11 \\
\hline
\end{tabular}

\section{Retraso de las Inversiones de la Primera Etapa (años) 2}

Fuente: contrato de concesión / Elaboración propia

\section{Causas que originan el problema en la entrega de terrenos}

Frente a este contexto de la demora en la entrega de terrenos desarrollado en el punto anterior, dentro de un proyecto donde las partes establecen los mecanismos de riesgos y adoptan las disposiciones para su mitigación, cabe preguntarse dónde se originó dicho problema. La respuesta es muy simple. Dicho problema se origina en el diseño del contrato de concesión ${ }^{11}$, debido a que el Estado peruano, con el objeto de impulsar las inversiones, ha elegido la política de encargar los proyectos sin tener necesariamente los estudios completos sobre la situación en que se encuentran las áreas de terrenos a ser entregados al concesionario para la ejecución de las obras, estableciendo fechas sin la seguridad de que se efectivicen.

11 Ya lo señalaba Luis Guasch (2005) al concluir en sus investigaciones, que las deficiencias de los contratos APP son básicamente el resultado de su diseño. Así, la probabilidad que se aceleren las renegociaciones se incrementan ante la problemática social, incidencias de la economía nacional e internacional, procesos apresurados, intereses creados y asesoramiento cuestionable, las limitaciones en cuanto a experiencia y recursos de los gobiernos en materia de diseño del contrato, que hacen que un concesionario quiera sacar provecho de la debilidad del Estado e imponer negociaciones a su favor (Engel y Ficher, 2008). Estos motivos pueden terminar en negociaciones no eficientes. 


\section{Antonio Lama More}

Asimismo, debe indicarse que el marco legal vigente de expropiaciones, en particular el marco legal para la liberación de terrenos, no obstante las diversas modificaciones, aún no permite actuar de manera eficaz para la entrega oportuna de los terrenos.

Sumado a ello, es sabido que el actuar del Estado es lento, burocrático y timorato de las acciones de control posterior, que lo hace en general ineficiente. En particular existe un problema de agencia para la entrega de terrenos, pues se observa que el Estado es el principal y a la vez el agente. Además no existen suficientes incentivos y penalidades a los actores del Estado para que realicen de manera oportuna y eficiente sus actividades, ya que ellos a la vez son juez y parte.

Bajo este contexto, se observa recurrentemente incumplimientos de entrega de terrenos en los plazos originalmente pactados por las partes (concedente-concesionario) en los proyectos de carreteras concesionadas, como el observado en el contrato de concesión de la Red Vial $N^{\circ}$ 06. Dichos incumplimientos tienen como principal efecto el retraso de las inversiones en las oportunidades establecidas en los contratos de concesión, con los consiguientes impactos en costos y disminución de su rentabilidad financiera y social.

\section{El problema en la entrega de terrenos y su efecto en el costo de oportuni- dad de los usuarios}

El problema de no entregar oportunamente los terrenos necesarios para la ejecución de las inversiones cobra mayor importancia debido a que dicha entrega está estrechamente vinculada a la culminación oportuna de las obras y, como se apreció para el caso de la Red Vial $N^{\circ} 06$, dicho retraso fue de 2 años.

Al respecto, es obvio que el atraso de la culminación de obras también retrasa los beneficios que los usuarios perciben de la ejecución de dichas obras en términos de:

- ahorro en tiempos de viaje, los cuales se pueden medir en términos de costo de oportunidad, ya que cada minuto de retraso en el viaje que experimentan los usuarios es la pérdida de un minuto que puede ser utilizado a generar ingresos y no afectar así su bienestar. 


\section{Pensamiento Crítico Vol. 19. N I}

- disminución de accidentes, ya que al contar con una infraestructura de calidad y con la totalidad de las obras ejecutadas (intercambios viales, pasos a desnivel, autopista) la seguridad de la vía aumenta y disminuye la probabilidad de accidente.

- menor costo operativo vehicular (COV), al contar con la totalidad de las obras ejecutadas los vehículos gastan menos combustible por realizar el viaje en un menor tiempo, el desgaste de los neumáticos es más lento, así como el mantenimiento del vehículo.

- entre otros.

Como se aprecia, el efecto último del problema de entrega oportuna de los terrenos tiene un efecto negativo sobre el bienestar de los usuarios, lo cual es preocupante ya que no se está cumpliendo oportunamente el objeto de haber concesionado el proyecto, el cual es brindar una infraestructura de servicio público de calidad, en este caso brindar una infraestructura de carretera, y en los tiempos establecidos en los contratos, que para el caso de la Red Vial № 06, era previsto tener la primera etapa ejecutada en su totalidad en septiembre del 2009, pero recién se obtuvo en septiembre del 2011.

Evidentemente resulta necesario estimar el efecto que tiene el retraso de la entrega de los terrenos en el bienestar de los usuarios, para cuyo efecto se ha estructurado un modelo financiero en el cual se determina el ahorro en tiempo que experimentarían los usuarios, obtenido de la diferencia entre el tiempo de viaje que toma recorrer la vía correspondiente a la primera con la totalidad de las obras ejecutadas (con autopista) y sin contar con la totalidad de las obras (Sin autopista)

Para ello se ha tomado los parámetros y datos que dispone el Sistema Nacional de Inversión Pública (SNIP) para ser tenidos en consideración por los PIP, referidos a los costos de oportunidad de los usuarios, el número de vehículos que circularon por la Red $N^{\circ} 06$, considerando solo el flujo vehicular del peaje de Chilca, debido a su cercanía con el tramo de la vía correspondiente a la primera etapa, así como las velocidades de viaje de un vehículo cuando existe autopista ( cuando se termina todas las obras de la primera etapa) y sin autopista ( cuando aún no se termina la primera etapa). En el siguiente cuadro se presenta dichos datos. 


\section{Antonio Lama More}

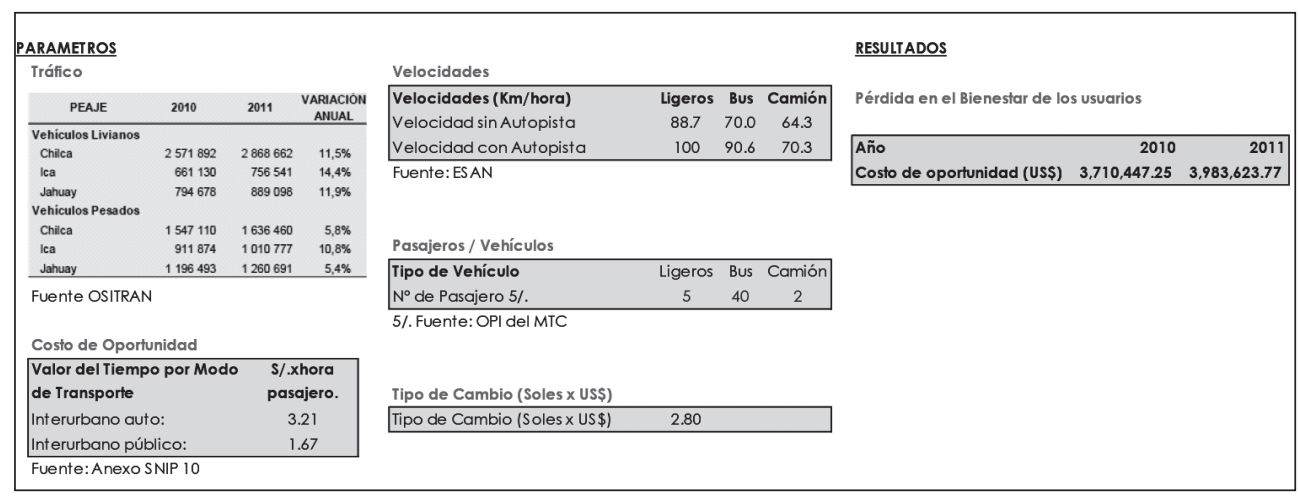

Con los datos antes mostrados y de acuerdo del modelo financiero se estimó la incidencia que sufrieron los usuarios en los dos años de retrasos de la inversión de la primera etapa, la cual para el año 2010 corresponde a US\$ 3.7 millones de dólares y para el año 2011 US $\$ 3.9$ millones de dólares. El detalle de los cálculos se muestra en el anexo del presente artículo.

Dichos montos que inciden directamente en los usuarios de la vía, no son compensados de manera directa y, por lo tanto, inciden en mejor y mayor bienestar de él y sus familias, así como los impactos de la sociedad que es afectada y que merece un análisis más específico.

\section{Conclusiones y recomendaciones}

- El problema de la demora en entregar áreas liberadas establecidas como necesarias en los proyectos de APP trae como consecuencia el retraso de las inversiones y su inevitable afectación en el bienestar de los usuarios.

- Dicho problema se origina esencialmente en el diseño del contrato de concesión, debido a que el estado peruano, con el objeto de impulsar las inversiones, ha elegido la política encargar los proyectos sin tener los estudios sobre la situación en que se encuentran las áreas de terrenos a ser entregados al concesionario para la ejecución de las Obras, tal como se evidenció en el contrato de concesión de la Red Vial $N^{\circ} 06$ y que sucede casi en la totalidad de los contratos de este tipo. 


\section{Pensamiento Crítico Vol. 19. N I}

- Se recomienda que el Estado impulse un marco legal más ágil y eficiente que permita que los procesos de liberación sean más eficaces y oportunos. E igualmente que los órganos promotores se aseguren de la disponibilidad de las áreas a ser entregadas en la concesión y, en el extremo de resultar necesario, que la liberación de áreas de los proyectos de APP sea de responsabilidad del titular del sector/región/gobierno local, en la oportunidad establecida en los respectivos contratos.

\section{Referencias bibliográficas}

Balcázar, C. (2003), "La Experiencia en Concesiones de Carreteras en el Perú y la Agenda Pendiente". PROINVERSION.

Edlin, A. and Hermalin, E. (2000), "Contract Renegotiation and Options in Agency Problems" Oxford University Press.

Engel, E.; Fischer, R.; Galetovic, A.; Hermosilla, M. (2008). "Renegociación de concesiones en Chile"

Estache, A. and Quesada, L. (2001) "Concession Contracts Renegotiation: The Efficiency and Equity Dilemma”. Policy Research Working Paper Nº 2705.

Guasch, J. L. (2005). "Concesiones en infraestructura. Cómo hacerlo bien”. Barcelona, Antoni Bosch

Ministerio de Transporte y Comunicaciones, "Contratos de Concesión en Transportes".

The Economist. Economist Intelligence Unit "Evaluando el entorno para las asociaciones público privadas en América Latina y el Caribe". Infrascope 2012.

PROINVERSIÓN (2005). Contrato de Concesión. CONCESIÓN DEL TRAMO VIAL PUENTE PUCUSANA - CERRO AZUL - ICA (RED VIAL 6). 


\section{Antonio Lama More}

\section{Anexo}

\section{Longitud de la 1 Etapa (km) $\quad 54.40$}

Velocidades
\begin{tabular}{|l|c|c|c|}
\hline Velocidades (Km/h) & Sin Autopista & Con Autopista & Velocidad \\
& A & B A & 11.30 \\
\hline Veh Ligeros (auto, SW, PickUp, Panel, Combi) & 88.70 & 100.00 & 20.60 \\
Buses & 70.00 & 90.60 & 6.00 \\
\hline Camiones(Camión, Semi-Trayler, Tryler) & 64.30 & 70.30 & \\
\hline
\end{tabular}

Fuente: ESAN

Estimación de redución del tiempo de viaje por la ejecución de las Obras de la 2E

\begin{tabular}{|l|c|c|c|}
\hline Tiempos de viaje por tipo de vehículo (minutos) & $\begin{array}{c}\text { Sin Autopista } \\
\text { C }\end{array}$ & $\begin{array}{c}\text { Con Autopista } \\
\text { D }\end{array}$ & $\begin{array}{c}\text { Tiempo } \\
\text { C-D }\end{array}$ \\
\hline Veh Ligeros (auto, SW, PickUp, Panel, Combi) & 36.80 & 32.64 & 4 \\
Buses & 46.63 & 36.03 & 11 \\
Camiones(Camión, Semi-Trayler, Tryler) & 50.76 & 46.43 & 4 \\
\hline
\end{tabular}

Estimación de los beneficios de los usuarios derivados del ahorro en tiempo de viaje

\begin{tabular}{|l|c|c|c|c|}
\hline Beneficio & $N^{\circ}$ pasajeros & Tiempo (h) & Valor del tiempo & Benef /veh (US\$) \\
\hline Veh Ligeros (auto, SW, PickUp, Panel, Combi) & 5 & 0.07 & 1.15 & 0.4 \\
Buses & 40 & 0.18 & 0.60 & 4.2 \\
Camiones (Camión, Semi-Trayler, Tryler) & 2 & 0.07 & 0.60 & 0.1 \\
\hline
\end{tabular}

Flujo de Beneficios Sociales

\begin{tabular}{|lrr|}
\hline Año Calendario & 2010 & 2011 \\
\hline Número de vehículos & & \\
\hline Vehi.Ligeros & $2,571,892$ & $2,868,662$ \\
Buses & 618,844 & 654,584 \\
Camiones & 928,266 & 981,876 \\
Total & $\mathbf{4 , 1 1 9 , 0 0 2}$ & $\mathbf{4 , 5 0 5 , 1 2 2}$ \\
& & \\
Perdida en el Costo de oportunidad & & \\
Vehi.Ligeros & $1,021,700$ & $1,139,594$ \\
Buses & $2,608,792$ & $2,759,457$ \\
Camiones & 79,955 & 84,572 \\
Total & $\mathbf{3 , 7 1 0 , 4 4 7}$ & $\mathbf{3 , 9 8 3 , 6 2 4}$ \\
\hline
\end{tabular}

